\title{
Organic Advertising? The Effect of Featured Review on Consumer Rating
}

\author{
Tong (Torrie) $\mathrm{Wu}$ \\ Zarb School of Business \\ Hofstra University \\ tong.wu@hofstra.edu
}

\author{
Abraham Seidmann \\ Simon Business School \\ University of Rochester \\ avi.seidmann@simon.rochester.edu
}

\author{
Huaxia Rui \\ Simon Business School \\ University of Rochester \\ huaxia.rui@simon.rochester.edu
}

\begin{abstract}
We investigate the effect of the Favorite Reviews (FR) feature on user behavior in an online usergenerated content (UGC) platform. The FR feature, which allows business owners to pin one selected review near the top of their property page, can serve as a new advertising tool for business owners to promote their businesses using satisfied consumers. Specifically, this paper investigates the impact of such a feature on the quantity of new reviews on TripAdvisor.com. Relying on difference-in-differences strategies, we find that this feature distorts the distribution of new reviews toward a positive extreme. The observed growth in new fivebubble reviews is mainly driven by new users. The experienced users, however, reduce their contributions, on average. This observed effect might result in user concern over the credibility of the UGC platform.
\end{abstract}

\section{Introduction}

In the current business world, paid advertising is a common tool allowing business owners to manipulate content and target ads to specific groups. Paid advertising can utilize many strategies. For example, celebrity endorsement is a common advertising strategy that has appeared in 10\% of US magazine ads [1]. Firms can decide who they would like to hire to endorse their products, but consumers perceive advertising as more manipulative than informative [2]. Consequently, the credibility of paid advertising may be questioned by consumers because business owners will only pick positive keywords meant to help maintain a positive brand image.

Online UGC has played an essential role in consumer purchase decisions in recent years. For example, eMarketer report [3] reveals that 61 percent of consumers consulted online reviews, blogs, and other forms of online customer feedback before making purchase decisions. For the travel and leisure industry, the number is more interesting. According to a survey by TurnTo about UGC and the commerce experience [4], 90 percent of around a thousand US consumers report UGC to be the most influential factor in their purchase decisions. This ratio is higher than those found with search engines and traditional advertisements. Indeed, consumers have become increasingly habitual in checking online UGC sites before making choices, ranging from what to eat for dinner [5], to which book to buy [6], to where to stay during vacation $[7,8]$.

One of the benefits of this type of organic advertising is that the online reviews are relatively more trustworthy than paid advertising because the content is from consumers. According to the Nielsen Global Trust in Advertising report [9], 92 percent of consumers trust organic UGC more than other forms of traditional advertising.

However, without manipulation, business owners can hardly control the contexts in which users are willing to share with the public. If the reviews are positive, the advertising could help create a positive image of their business. However, if the reviews are negative the business may lose potential consumers due to the intense competition from similar companies. Hence, the promotional benefit is smaller when compared with paid advertising.

Recently, the emergence of the "Favorite Reviews" (FR) feature on UGC platforms has unlocked the possibility of a new type of advertising, which falls somewhere between paid advertising and organic advertising. On the one hand, this special feature allows business owners to select one review that can best represent their businesses and present it on the profile page, allowing business owners to promote their businesses through an endorsement from a consumer. Since the review is from a consumer, the content seems more credible than paid advertising. On the other hand, the cost of this FR feature is relatively low to business owners versus traditional advertising (or totally free). For example, back in 2009, Yelp allowed business owners to pin a "favorite review" on top of other reviews. Unfortunately, Yelp eliminated this feature in 2010 due to a lawsuit that was caused by a concern over "manipulation." However, some UGC platforms, such 
as Glassdoor and TripAdvisor, still allow business owners to pick a featured review. Glassdoor pins an employer's featured review on top of other reviews, like Yelp's favorite review in 2010, whereas TripAdvisor lets an accommodation pin its favorite review in the second slot.

The FR feature can help consumers easily discover the best part of a business or a product. This feature can, to some extent, reduce the searching cost for potential consumers who want to know the best parts of a hotel. However, if the FR feature is really that "great," why did Yelp decide to remove it a few years ago? To the best of our knowledge, no study has investigated the impact of this special feature on consumer behavior or on the UGC platform. Our paper aims to fill in the gaps by answering the following questions: Can the FR feature promote online word-of-mouth (WOM), that is, can we expect more user reviews after launching the FR for a hotel? Rational hotel owners or managers will pick a positive review as favorite review. Would this encourage consumers to write more positive reviews? The answers to these questions are important for UGC platforms and hotels. Platforms like TripAdvisor need to investigate whether the FR feature can promote content contribution without sabotaging their relationships with consumers. Hotels need to find out whether the FR feature can be an effective reputational management tool, which can bring more active customers. Powerful reputation management can generate a more positive evaluation of a hotel [10]. Most importantly, users may get motivated after seeing favorite reviews and thus write more high-quality reviews. Those users whose reviews are chosen as FR may exert more effort in contributing content to the community.

Using a difference-in-differences technique, we find significant and robust increases in the volume of new five-bubble reviews for the hotels that have implemented the FR feature, on average, as compared with a matched control group. The results imply that the distribution of new reviews is distorted toward a positive extreme. We also find that the incremental fivebubble reviews are mainly driven by the new users rather than the experienced users on the platform. The experienced users, however, reduce their contributions, on average.

The research makes two important contributions to the literature. First, to the best of our knowledge, our research is the first that empirically investigates the effect of the FR feature on quantity of new reviews on a UGC platform. We find evidence that this type of feature could be an effective reputation management tool for business owners. But this feature may bring some concerns regarding the credibility of reviews on the UGC platform in the short run. Second, our research also sheds light on the motivations of users to contribute content to a UGC platform.

\section{Hypothesis}

Not surprisingly, most of the favorite reviews are reviews with high ratings. In our sample of 527 initial favorite reviews, 515 of them have five-bubble rating with the rest having four-bubble rating. We hypothesize that displaying these highly rated favorite reviews at prominent positions will affect future reviewers' rating decision.

First, most reviewers are likely to be more motivated to contribute high-rating reviews. Content contribution can stem from social psychology theory. In selfdetermination theory (SDT), Ryan and Deci [11] define two distinct types of motivations: intrinsic and extrinsic. Intrinsic motivation refers to motivation driven by inherent satisfaction, because the action is naturally interesting or enjoyable, such as helping the community, rather than the appeal of external rewards or punishments [11]. By contrast, extrinsic motivation is defined as an action taken due to external reasons or outcomes, such as economic rewards or non-economic rewards like public recognition [12]. Although economic rewards have been identified as a driver of eWOM behavior, as proven by Khern-am-nuai et al. [13] and Qiao et al. [14], nonfinancial rewards are more common for UGC platforms. Research has shown that psychological rewards like badges, reputation $[15,16$, 17], perceived identity verification [18], informal recognition [19], and social comparison [20] are all extrinsic motivators for content contribution.

Badges can motivate users to increase their level of participation, and this gamification element has been used extensively in online UGC sites. Similar to badges, the joy of having a review chosen as a hotel's favorite is an attractive prospect users can pursue, potentially incentivizing other users to contribute more five-bubble reviews. Moreover, the FR feature is more salient than badges for users who read the review content. Therefore, we believe that the "recognition" created by the FR feature may increase the reputation of the FR reviewers, which is a crucial factor of information sharing behavior [16]. If a consumer desires to gain fame in an online community, she has a higher tendency to spread eWOM. The FR feature would serve as a reputation-boosting strategy that encourages content contribution. Since the favorite review will be pinned at the top slots, the content would be seen by a lot more users than other reviews. Hence, a reviewer's reputation would be enhanced if her review is chosen as the favorite review. As a result, this kind of social recognition with a symbolic reward might encourage users to write more five-bubble reviews. More 
importantly, the desire to become a "Favorite Reviews" reviewer may push other users who have never written a review to start contributing five-bubble reviews. These users may do so with the hope of being chosen as the favorite review in order to gain reputation, while in the meantime, returning a favor to the nice hotel owner.

Second, anchoring effect may be another mechanism through which reviewers' rating decisions could be affected by the implementation of the FR feature. According to Tversky and Kahneman [21], anchoring represents a heuristic by which biased decisions are made based on an initially presented value. Following these authors, a number of studies have shown that anchoring effect exists in decision making process [22]. When the anchoring information is more accessible, that information is more likely to become a starting point in an individual's decision [23]. For example, reviewers tend to anchor on prior reviews to make their own evaluation [24]. Since a favorite review is pinned on the second top slot, the rating and content are salient. Hence, it is highly possible that users would anchor on the bubble rating of the favorite review and write their reviews. Since favorite reviews tend to be five-bubble reviews, the volume of new five-bubble reviews would increase after the FR feature is implemented.

Finally, social comparison could be another motivation that can make users contribute when the FR feature is present. The theory suggests that individuals are prone to evaluate their capabilities and perspectives in comparison with others [25]. Individuals tend to compare themselves to those with better status, capability, and performance for the purposes of selfimprovement [26]. This is because upward social comparison can be motivational [27, 28]. Observing other people who perform better would make individuals establish higher standards, exert more effort, and enhance their own performance [29]. A reviewer is more likely to write a five-bubble review after seeing the pinned favorite review of a hotel, because the current chosen favorite is expected to be a high-quality review with a high score that can serve as a "role model" for the new positive reviews. If a user is satisfied with a hotel, it is very likely that she will return a favor to the hotel. Therefore, users tend to write high-quality, positive reviews in order to be noticed by the business owners.

To summarize, we propose the following hypothesis for empirical testing.

Hypothesis: The Favorite Reviews feature positively affects the volume of high-rating reviews.

\section{Data}

Online UGC has been particularly important in the travel sector. We collected information related to reviews from Tripadvisor.com, a US-based UGC platform that provides reviews of travel-related content. Tripadvisor is also the world's largest travel community (https://tripadvisor.mediaroom.com/us-about-us).

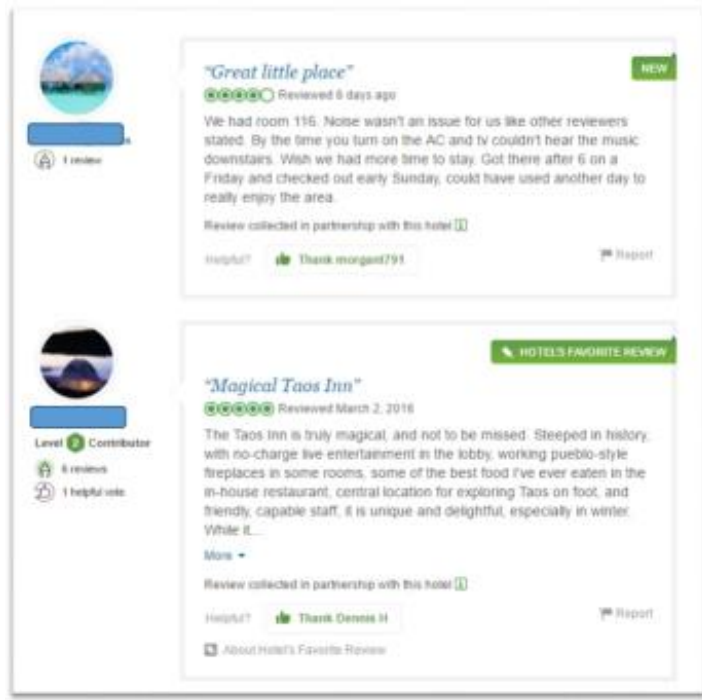

Figure 1. Example of the Hotel's Favorite Reviews Feature on TripAdvisor.com

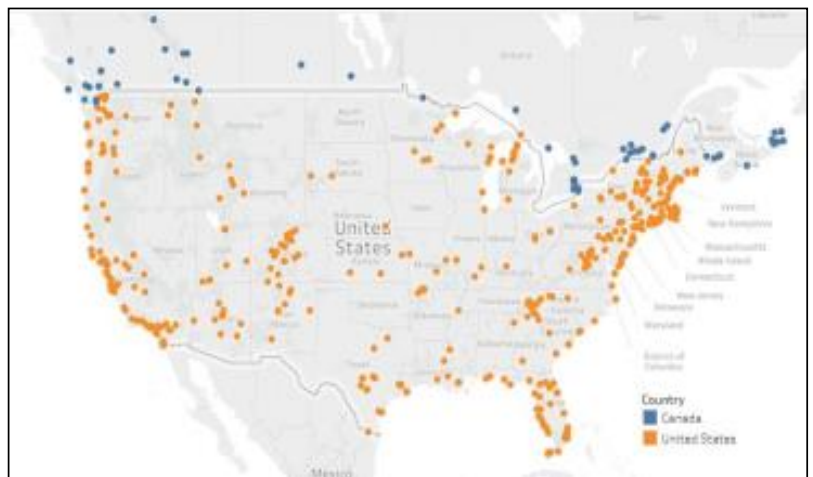

Figure 2. Geographic Distribution of Focal Hotels

Starting from May 2016, TripAdvisor implemented a new feature, Favorite Reviews (FR), on randomly selected accommodations. The assignment of the FR feature was offered to hotels upon their business listing subscription renewal. Since the date of contract renewal is random among hotels, the assignment of this feature is exogenous. Figure 1 depicts an example of this FR feature. We refer to those accommodations that launched the FR feature as "focal hotels" for simplicity throughout this paper. To ensure a more homogenous sample, we focus on the independent focal hotels in the United States and Canada only. We closely monitored the hotels that launched the FR feature May $10^{\text {th }}, 2016$ to September $30^{\text {th }}, 2016$. For each focal hotel, we also 
collected data for similar hotels that are shown on each hotel's landing page. Those hotels were labeled as "Hotels you might also like..." and were recommended by Tripadvisor.com. There were up to six hotels near the location of the focal hotel. Finally, after excluding the overlapping hotels and hotels with missing data on key variables, we ended up with 527 focal hotels and 2210 matching control hotels. Figure 2 displays the geographic distributions of those focal hotels.

Our final data set includes review data for these hotels from March 2016 to October 2016. For each hotel, we collected the following information: hotel's ID, country, type, date of implementation of the FR feature, FR's ID, star level, number of rooms, average price, special offer, business listing, total number of reviews, and bubble rating on TripAdvisor. For the reviews of a hotel, we collected each review's ID, date of posting, bubble rating, reviewer's ID, and the review content. In addition, we also collected users' entire review history. Table 1 shows the summary statistics for focal and control hotels from March to October.

Table 1. Summary Statistics for Focal and Control Hotels (Average over Mar-Oct)

\begin{tabular}{rrlrrrr}
\hline Type & Count & Price & Rating & \# Rooms & Star Level & \# Reviews \\
\hline Focal & 527 & 226.46 & 4.41 & 51 & 2.33 & 307 \\
Control & 2210 & 208.99 & 4.18 & 101 & 2.89 & 520 \\
\hline
\end{tabular}

Our panel data is unbalanced due to a combination of different FR feature implementation dates. To reduce concerns that this might bias our results, we set the same "cut-off" date for the control hotels as their matching focal hotels. We also excluded the week when the FR feature was implemented.

Table 2. Descriptive Statistics for WeekLevel Samples of Hotel Variables from March to October 2016 (week 10 to week 43)

\begin{tabular}{lcrrrr}
\hline \multicolumn{1}{c}{ Variable } & Obs & Mean & Std. Dev. & Min & \multicolumn{1}{c}{ Max } \\
\hline Focal & 90,001 & 0.19 & 0.39 & 0 & 1 \\
Num_total & 90,001 & 2.65 & 4.18 & 0 & 98 \\
Num_5 & 90,001 & 1.50 & 2.68 & 0 & 54 \\
Num_4 & 90,001 & 0.68 & 1.33 & 0 & 33 \\
Num_3 & 90,001 & 0.27 & 0.72 & 0 & 20 \\
Num_2 & 90,001 & 0.11 & 0.40 & 0 & 10 \\
Num_1 & 90,001 & 0.08 & 0.32 & 0 & 6 \\
Stddev & 90,001 & 0.79 & 1.13 & 0 & 24.04 \\
Avg_wecklyrating & 60,714 & 4.26 & 0.81 & 1 & 5 \\
\hline
\end{tabular}

Note. Focal is a dummy variable indicating that the FR feature is implemented. Num total represents the total number of new reviews per week. Num_5, Num_4, Num_3, Num_2, and Num_1 represent the total number of new five-, four-, three-, two-, and one-bubble reviews per week respectively. Stddev is the sample standard deviation based on the distribution of review volumes with different bubble levels per week. Avg.weeklyrating is the average bubble rating per week.
The variables of interest are the volumes of new reviews at each bubble level. Particular attention is paid to the number of extremely positive reviews (i.e., fivebubble reviews) and very negative reviews (i.e., one- or two-bubble reviews). We aggregate the number of new reviews by each consecutive seven-day period starting from January 1, 2016 and simply call each period a week. We designate the first full week after implementation as the first treatment week. Table 2 summarizes the descriptive statistics of our week-level samples of hotel variables for whole weeks between March and October 2016.

\section{Identification Strategy}

Since the FR feature is free and it can serve as a free advertisement to show the best parts of a hotel, we can assume that each focal hotel that was offered the feature would implement it immediately. Since each focal hotel is randomly offered to implement the feature, the assignment of the FR feature can be considered as a random event. Therefore, we can use a difference-indifferences technique to study the impact of the implementation of the FR feature on the volume of reviews at each bubble level. Panel data structure allows us to use hotel-level fixed effects to control for timeinvariant unobserved heterogeneity.

The general econometric model is:

$$
\begin{gathered}
Y_{i t} \\
=\beta_{0}+\beta_{1} \text { Focal }_{i} \times \text { AfterFR } \\
+\alpha_{i}+\theta_{t}+\varepsilon_{2} \text { AfterFR } R_{i t}+\beta_{3} X_{i t}
\end{gathered}
$$

Where $Y_{i t}$ is the dependent variable for hotel $i$ in week $t . \alpha_{i}$ is the set of hotel fixed effects to account for unobserved heterogeneity for each hotel, $\theta_{t}$ is a set of dummy variables for each week. Focal $i$ is a dummy variable equal to 1 if a hotel is in the focal group, and 0 if it is a control hotel.AfterF $R_{i t}$ is a time-varying dummy variable equal to 1 for all week $t$ at hotel $i$ after launching the FR feature. $X_{i t}$ represents the observed time-varying factors for each hotel $i$ at week $t$. The coefficient $\beta_{1}$ measures the DD effect.

\section{Empirical Results}

If the distribution of reviews has been shifted to the more extreme side after launching the FR reviews, we would expect an increase in the quantity of new fivebubble reviews and possibly a decrease of the number of other bubble-level reviews for focal hotels as compared with control hotels. Without making any 
assumptions about the model specification, we plot and test the model-free distribution of the volume of reviews at each bubble-level before and after the FR feature for focal and control hotels, as shown in Figure 3. We divide the sample into four sub-samples for focal and control hotels before and after the FR feature for each week. In each sub-sample, we compute the proportion of new reviews at each bubble level and indicate the $95 \%$ confidence bands on each bar. The non-parametric Wilcoxon rank-sum tests are used to confirm what we see from the figure.

The model-free distributions of new reviews indicate that the proportion of new five-bubble reviews among all reviews increase significantly after the implementation of the FR feature for focal hotels as compared with control hotels, while the proportions of new three- and four-bubble reviews decrease significantly relative to control hotels (p-value $<0.05$ ) after the FR feature is implemented. The proportion of one-bubble reviews also decreases after the FR feature for the control group at $10 \%$ level based on the Wilcoxon rank-sum test.

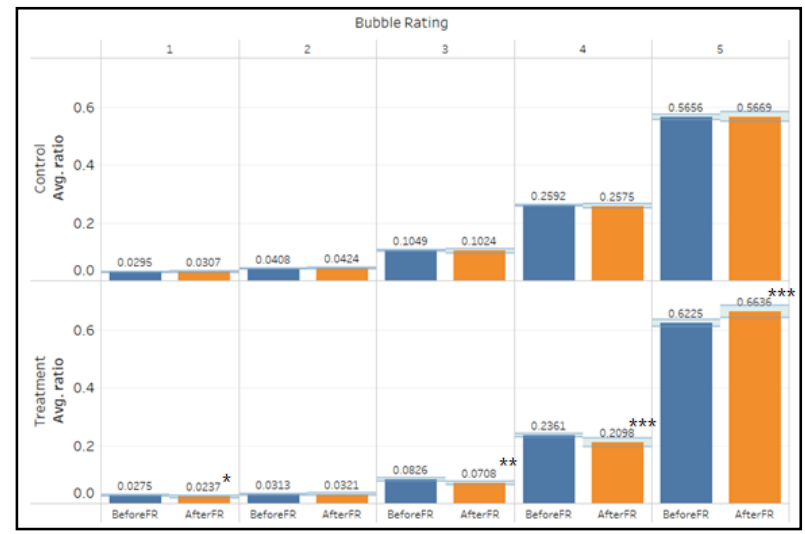

Note. We divide the full sample into four sub-samples for each week: beforeFR/control, beforeFR/treatment, afterFR/control, and afterFR/treatment. The proportions of the volumes of new reviews at different bubble levels are also computed to reflect the distribution of new reviews for each subsample. Each bar presents the average proportion for each category with 95\% confidence bands.

Figure 3. Model-free Distribution of Number of Reviews

Next, we study the impact of the FR feature on the volume of new five-bubble reviews with the help of linear fixed effect models. Our specification exploits the panel nature of the data by incorporating a full set of fixed effects to control for the unobserved heterogeneity.

Our results reported in Table 3 suggest that the implementation of the FR feature leads to an increment of weekly new five-bubble reviews relative to the control group. The models include hotel fixed effects that account for time-invariant hotel homogeneity, as well as week fixed effects to control for time trends. Columns (1) and (2) present the results based on linear fixed effect models. Column (1) shows that implementation of the FR feature increases the number of new five-bubble reviews. In terms of effect sizes, compared with the average weekly volume of new fivebubble reviews in the sample (mean $=1.5)$, the FR feature increases five-bubble review quantity by 0.1 (6.7\%) per week on average. In Column (2), we include a few more time-varying variables, including the bubble rating for a hotel for the previous week, total number of posted reviews until the previous week, and the number of special offers posted in the prior week. We find that the estimated coefficient of the DD term in Column (2) is still significant and positive with a similar magnitude as Column (1). The findings suggest that the FR feature results in a significant increase in the contribution of five-bubble reviews on a weekly basis, supporting our Hypothesis.

Table 3. Impact of FR on Number of New Five-bubble Reviews (Weekly)

\begin{tabular}{lll}
\hline & $(1)$ & $(2)$ \\
\hline & Linear FE & Linear FE \\
\hline AfterFR & -0.0348 & -0.0307 \\
& $(0.0309)$ & $(0.0310)$ \\
Focal $\times$ AfterFR & $0.1005^{* * *}$ & $0.0900^{* *}$ \\
& $(0.0399)$ & $(0.0404)$ \\
Rating_lagl & & 0.1208 \\
& & $(0.0807)$ \\
TotalReview_lagl & & -0.0004 \\
& & $(0.0004)$ \\
SpecialOffer_lagl & & $0.0073^{*}$ \\
& & $(0.0040)$ \\
Week fixed effect & controlled & controlled \\
Hotel fixed effect & controlled & controlled \\
Constant & $1.0326^{* * * *}$ & $0.7072^{*}$ \\
& $(0.0293)$ & $(0.3705)$ \\
\hline $\mathrm{N}$ & 90001 & 89623 \\
$\mathrm{R}^{2}$ & 0.6831 & 0.6849 \\
\hline
\end{tabular}

Note. The dependent variable is Num_5, representing the total number of new five-bubble reviews per week for Columns (1) and (2). Focal is a dummy variable that equals 1 if a hotel is in the focal group, and 0 if it is a control hotel. Rating_lag1 is the highest bubble rating score on a hotel's profile page for the previous week. TotalReview_lag1 refers to the total number of reviews on a hotel's profile page for the previous week. Specialoffer_lag1 represents the number of times a special offer is posted on a hotel's profile page for the previous week.

Standard errors clustered at the hotel level in parentheses. ${ }^{*} p<0.1,{ }^{* *} p<0.05$, and ${ }^{* * *} p<0.01$.

Next, we apply the same econometric models to explore the impact of the FR feature on the total number 
of new reviews, as well as the volume of new badreviews, which include one- and two-bubble reviews.

Table 4 reports the findings for total volume. The results on Columns (1) and (2) reveal that the volume of new reviews does not significantly increase for the focal hotels relative to the control hotels after implementation of the FR feature. Our results suggest that distribution of reviews shifts to a positive extreme.

Table 4. Impact of FR on Number of New Reviews (Weekly)

\begin{tabular}{lll}
\hline & $(1)$ & $(2)$ \\
\hline & Linear FE & Linear FE \\
\hline AfterFR & -0.0328 & -0.0243 \\
& $(0.0509)$ & $(0.0509)$ \\
Focal $\times$ AfterFR & 0.0526 & 0.0189 \\
& $(0.0576)$ & $(0.0611)$ \\
Rating_lagl & & $0.4269^{* * *}$ \\
& & $(0.1275)$ \\
TotalReview_lagl & & -0.0011 \\
& & $(0.0009)$ \\
SpecialOffer_lagl & & $0.0143^{* *}$ \\
& & $(0.0058)$ \\
Week fixed effect & controlled & controlled \\
Hotel fixed effect & controlled & controlled \\
Constant & $1.7481^{* * *}$ & 0.4237 \\
& $(0.0445)$ & $(0.6017)$ \\
\hline $\mathrm{N}$ & 90001 & 89623 \\
$\mathrm{R}^{2}$ & 0.7348 & 0.7364 \\
\hline
\end{tabular}

Note. The dependent variable is Num_total, representing the total number of new reviews per week for Columns (1) and (2). Focal is a dummy variable that equals 1 if a hotel is in the focal group, and 0 if it is a control hotel. Rating_lag1 is the highest bubble rating score on a hotel's profile page for the previous week. TotalReview_lag1 refers to the total number of reviews on a hotel's profile page for the previous week. Specialoffer_lag1 represents the number of times a special offer is posted on a hotel's profile page for the previous week.

Standard errors clustered at the hotel level in parentheses. ${ }^{*} p<0.1,{ }^{* *} p<0.05$, and ${ }^{* * *} p<0.01$.

Table 5. Impact of FR on Number of New Bad Reviews (Weekly)

\begin{tabular}{lcc}
\hline & $(1)$ & $(2)$ \\
\hline & Linear FE & Linear FE \\
\hline AfterFR & $0.0194^{* *}$ & $0.0203^{* *}$ \\
& $(0.0098)$ & $(0.0099)$ \\
Focal $\times$ AfterFR & $-0.0219^{* *}$ & $-0.0261^{* * *}$ \\
& $(0.0090)$ & $(0.0097)$ \\
Rating_lagl & & $0.0832^{* * *}$ \\
& & $(0.0224)$ \\
TotalReview_lagl & & -0.0001 \\
& & $(0.0001)$ \\
SpecialOffer_lagl & & 0.0005 \\
& & $(0.0009)$ \\
Week fixed effect & controlled & controlled \\
Hotel fixed effect & controlled & controlled \\
Constant & $0.1126 * * *$ & -0.1752 \\
& $(0.0078)$ & $(0.1084)$ \\
\hline $\mathrm{N}$ & 90001 & 89623 \\
$\mathrm{R}^{2}$ & 0.3312 & 0.3319 \\
\hline
\end{tabular}

Note. The dependent variable is Num_bad, which is the sum of Num_1 and Num_2, representing the total number of new bad reviews per week for Columns (1) and (2). Focal is a dummy variable that equals 1 if a hotel is in the focal group, and 0 if it is a control hotel. Rating_lag1 is the highest bubble rating score on a hotel's profile page for the previous week. TotalReview_lag1 refers to the total number of reviews on a hotel's profile page for the previous week. Specialoffer_lag1 represents the number of times a special offer is posted on a hotel's profile page for the previous week.

Standard errors clustered at the hotel level in parentheses. ${ }^{*} p<0.1,{ }^{* *} p<0.05$, and ${ }^{* * *} p<0.01$.

Fixed effect regression models show that the estimated coefficients of the DD terms are significantly negative. The result, as illustrated in Table 5, indicates that the number of bad reviews decreases after implementation of the FR feature. The estimated coefficient of DD term in Column (1) suggests that, on average, the volume of bad reviews decreases by $11.5 \%$ after the FR feature is implemented for focal hotels.

\section{Robustness Check}

We conduct additional tests to further check the robustness of our results.

First, we test whether there exists any pre-trend, and how the effect unfolds over time. Specifically, AfterFR_lead3 equals 1 if the week is 3 weeks before adoption for the focal hotel, and 0 otherwise; AfterFR_lead 2 equals 1 if the week is 2 weeks before adoption for the focal hotel, and 0 otherwise; AfterFR_leadl is equal to 1 if the week is the week right before adoption for the focal hotel, and 0 otherwise; AfterFR_lagl equals 1 if the week is the week right after adoption for the focal hotel, and 0 otherwise; AfterFR_lag2+ equals 1 if the week is the second, the third week, or more weeks after adoption for the focal 
hotel, and 0 otherwise. We use 4 or more weeks before adoption as the benchmark. Tables 6 depicts the results when dependent variable is the number of new fivebubble reviews.

The results from Table 6 show that the pre-adoption trends between focal and control hotels are the same for Num_5. The coefficients of lags indicate that the impact of FR feature on the number of new five-bubble reviews fades out over time.

Finally, we aggregate the number of reviews by month and carry out the same regression analyses. Our preliminary results show that the impact of the FR feature on the volumes of new five-bubble and bad reviews is consistent with the main models. For the baseline model, the magnitude of the coefficient of the DD term is about 4.7 times larger than the one found from the weekly analysis. The monthly level analysis also shows that the impact of the FR feature on the volume of new bad reviews is significantly negative when the first two fixed effect models are used. The size of the coefficient on DD term is about 4.9 times larger than the one we obtain by weekly analysis.

Table 6. Effect of Timing on Num_5

\begin{tabular}{lll}
\hline & \multicolumn{1}{c}{$(1)$} & \multicolumn{1}{c}{$(2)$} \\
\hline & Linear FE & Linear FE \\
\hline AfterFR_lead3 & 0.0190 & 0.0191 \\
& $(0.0522)$ & $(0.0522)$ \\
AfterFR_lead2 & 0.0531 & 0.0542 \\
& $(0.0715)$ & $(0.0715)$ \\
AfterFR_lead1 & 0.1040 & $0.1058^{*}$ \\
& $(0.0640)$ & $(0.0640)$ \\
AfterFR_lagl & $0.1398^{* *}$ & $0.1347^{* *}$ \\
& $(0.0612)$ & $(0.0613)$ \\
AfterFR_lag2+ & $0.0927^{* *}$ & $0.0833^{* *}$ \\
& $(0.0418)$ & $(0.0421)$ \\
Rating_lagI & & 0.1213 \\
& & $(0.0808)$ \\
TotalReview_lagI & & -0.0004 \\
& & $(0.0004)$ \\
SpecialOffer_lag1 & & $0.0074 *$ \\
& & $(0.0040)$ \\
Week fixed effect & controlled & controlled \\
Hotel fixed effect & controlled & controlled \\
Constant & $1.0326 * * *$ & $0.7048^{*}$ \\
& $(0.0293)$ & $(0.3708)$ \\
\hline N & 90001 & 89623 \\
$\mathrm{R}^{2}$ & 0.6831 & 0.6849 \\
\hline
\end{tabular}

Note. The dependent variable is Num_5, representing the total number of new five-bubble reviews per week for Columns (1) and (2). AfterFR_lead3 equals 1 if the week is 3 weeks before adoption for the focal hotel, and 0 otherwise; AfterFR_lead2 equals 1 if the week is 2 weeks before adoption for the focal hotel, and 0 otherwise; AfterFR_leadl is equal to 1 if the week is the week right before adoption for the focal hotel, and 0 otherwise; AfterFR_lagl equals 1 if the week is the week right after adoption for the focal hotel, and 0 otherwise; AfterFR_lag2 + equals 1 if the week is the second, third week, or more after adoption for the focal hotel, and 0 otherwise. Rating_lag1 is the highest bubble rating score on a hotel's profile page for the previous week. TotalReview_lag1 refers to the total number of reviews on a hotel's profile page for the previous week. Specialoffer_lag1 represents the number of times a special offer is posted on a hotel's profile page for the previous week.

Standard errors clustered at the hotel level in parentheses. ${ }^{*} p<0.1,{ }^{* *} p<0.05$, and ${ }^{* \star *} p<0.01$.

\section{Heterogeneous Effect}

The main estimation results in the last two sections suggest that there is an incentive distortion which shifts the contribution of reviews toward a more positive extreme. In this section, we intend to find the underlying reasons why the volume of new five-bubble reviews increases but the number of new bad-reviews decreases after the FR. To do that, we split the number of new fivebubble reviews into two parts based on user type: new and experienced reviewer. If a review in our sample is the first review in a user's entire review history, we label that user as a new reviewer. In contrast, if a review in our sample is not her first review, we call that user an experienced reviewer. Then, we run regression models for the number of five-bubble reviews and bad-reviews created by new reviewers and experienced reviewers separately with the same specifications as our main models. Table 7 and Table 8 report the results for new reviewers and experienced reviewers, respectively, when the dependent variable is the volume of new fivebubble reviews. Tables 9 and 10 show the results for bad-reviews for new and experienced reviewers. To our surprise, the results show that the increment of new fivebubble reviews is driven majorly by new users, as the estimated coefficients of DD terms are significant only for the subsample with new users. Column (1) in Table 7 shows that new reviewers contribute for $85.3 \%$ of the effect of the FR on the volume of new five-bubble reviews. The reduction in bad reviews, however, is driven majorly by the experienced reviewers rather than the new reviewers. Column (1) in Table 9 shows that experienced reviewers contribute for $76.3 \%$ of the effect of the FR on the volume of new bad reviews.

Table 7. Impact of FR on Number of New Five-bubble Reviews by New Reviewers (Weekly) 


\begin{tabular}{lll}
\hline & $(1)$ & $(2)$ \\
\hline & Linear FE & Linear FE \\
\hline AfterFR & -0.0199 & -0.0161 \\
& $(0.0188)$ & $(0.0189)$ \\
Focal $\times$ AfterFR & $0.0853^{* * *}$ & $0.0724 * * *$ \\
& $(0.0227)$ & $(0.0230)$ \\
Rating_lag1 & & 0.0532 \\
& & $(0.0487)$ \\
TotalReview_lag1 & & -0.0004 \\
& & $(0.0003)$ \\
SpecialOffer_lag1 & & $0.0056^{* *}$ \\
& & $(0.0026)$ \\
Week fixed effect & controlled & controlled \\
Hotel fixed effect & controlled & controlled \\
Constant & $0.4912 * * *$ & $0.4431 *$ \\
& $(0.0196)$ & $(0.2419)$ \\
\hline $\mathrm{N}$ & 90001 & 89623 \\
$\mathrm{R}^{2}$ & 0.5896 & 0.5919 \\
\hline
\end{tabular}

Note. The dependent variable is Num_5New, representing the number of new five-bubble reviews written by new reviewers per week for Columns (1) and (2). Focal is a dummy variable that equals 1 if a hotel is in the focal group, and 0 if it is a control hotel. Rating_lag1 is the highest bubble rating score on a hotel's profile page for the previous week. TotalReview_lag1 refers to the total number of reviews on a hotel's profile page for the previous week. Specialoffer_lag1 represents the number of times a special offer is posted on a hotel's profile page for the previous week.

Standard errors clustered at the hotel level in parentheses. ${ }^{*} p<0.1,{ }^{* *} p<0.05$, and ${ }^{* * *} p<0.01$.

Table 8. Impact of FR on Number of New Five-bubble Reviews by Experienced Reviewers (Weekly)

\begin{tabular}{lll}
\hline & $(1)$ & $(2)$ \\
\hline & Linear FE & Linear FE \\
\hline AfterFR & -0.0148 & -0.0145 \\
& $(0.0192)$ & $(0.0191)$ \\
Focal $\times$ AfterFR & 0.0153 & 0.0178 \\
& $(0.0248)$ & $(0.0272)$ \\
Rating_lag1 & & 0.0676 \\
& & $(0.0431)$ \\
TotalReview_lag1 & & -0.0000 \\
& & $(0.0004)$ \\
SpecialOffer_lag1 & & 0.0016 \\
& & $(0.0023)$ \\
Weekfixed effect & controlled & controlled \\
Hotel fixed effect & controlled & controlled \\
Constant & $0.5414 * * *$ & 0.2642 \\
& $(0.0170)$ & $(0.2670)$ \\
\hline $\mathrm{N}$ & 90001 & 89623 \\
$\mathrm{R}^{2}$ & 0.5866 & 0.5875 \\
\hline
\end{tabular}

Note. The dependent variable is Num_5Exp, representing the number of new five-bubble reviews written by experienced reviewers per week for Columns (1) and (2). Focal is a dummy variable that equals 1 if a hotel is in the focal group, and 0 if it is a control hotel. Rating_lag1 is the highest bubble rating score on a hotel's profile page for the previous week. TotalReview_lag1 refers to the total number of reviews on a hotel's profile page for the previous week. Specialoffer_lag1 represents the number of times a special offer is posted on a hotel's profile page for the previous week.

Standard errors clustered at the hotel level in parentheses. ${ }^{*} p<0.1,{ }^{* *} p<0.05$, and ${ }^{* * *} p<0.01$.

Table 9. Impact of FR on Number of New Bad Reviews by New Reviewers (Weekly)

\begin{tabular}{lll}
\hline & $(1)$ & $(2)$ \\
\hline & Linear FE & Linear FE \\
\hline AfterFR & $0.0155^{* *}$ & $0.0159^{* *}$ \\
& $(0.0063)$ & $(0.0063)$ \\
Focal $\times$ AfterFR & $-0.0095^{*}$ & $-0.0102^{*}$ \\
& $(0.0056)$ & $(0.0056)$ \\
Rating_lag1 & & $0.0475^{* * *}$ \\
& & $(0.0144)$ \\
TotalReview_lag1 & & -0.0000 \\
& & $(0.0000)$ \\
SpecialOffer_lag1 & & -0.0001 \\
& & $(0.0005)$ \\
Week fixed effect & controlled & controlled \\
Hotel fixed effect & controlled & controlled \\
Constant & $0.0573^{* * *}$ & $-0.1259^{* *}$ \\
& $(0.0052)$ & $(0.0606)$ \\
\hline N & 90001 & 89623 \\
$\mathrm{R}^{2}$ & 0.2506 & 0.2512 \\
\hline
\end{tabular}

Note. The dependent variable is Num BadNew, representing the number of new five-bubble reviews written by experienced reviewers per week for Columns (1) and (2). Focal is a dummy variable that equals 1 if a hotel is in the focal group, and 0 if it is a control hotel. Rating_lag1 is the highest bubble rating score on a hotel's profile page for the previous week. TotalReview_lag1 refers to the total number of reviews on a hotel's profile page for the previous week. Specialoffer_lag1 represents the number of times a special offer is posted on a hotel's profile page for the previous week.

Standard errors clustered at the hotel level in parentheses. ${ }^{*} p<0.1,{ }^{* *} p<0.05$, and ${ }^{* * *} p<0.01$.

To check the robustness of the results above, we also split the reviews based on the time when a user joined the TripAdvisor platform as a member. The joining date is specified with the year and month. We separate the reviews by new users, who joined the platform within 30 days, and by old users, who joined the TripAdvisor community more than 30 days before a review is written. The results are consistent with our findings above when we split user reviews in a different way.

The findings imply that the incremental five-bubble reviews for the focal hotels might be fake reviews written by reviewers who might be associated with the business owners or managers after the FR is implemented. 
Table 10. Impact of FR on Number of New Bad Reviews by Experienced Reviewers (Weekly)

\begin{tabular}{lll}
\hline & $(1)$ & $(2)$ \\
\hline & Linear FE & Linear FE \\
\hline AfterFR & 0.0038 & 0.0044 \\
& $(0.0059)$ & $(0.0060)$ \\
Focal $\times$ AfterFR & $-0.0124^{* *}$ & $-0.0159^{* *}$ \\
& $(0.0057)$ & $(0.0065)$ \\
Rating_lag1 & & $0.0357^{* * *}$ \\
& & $(0.0134)$ \\
TotalReview_lag1 & & -0.0001 \\
& & $(0.0001)$ \\
SpecialOffer_lag1 & & 0.0006 \\
& & $(0.0006)$ \\
Week fixed effect & controlled & controlled \\
Hotel fixed effect & controlled & controlled \\
Constant & $0.0553 * * *$ & -0.0493 \\
& $(0.0049)$ & $(0.0777)$ \\
\hline $\mathrm{N}$ & 90001 & 89623 \\
$\mathrm{R}^{2}$ & 0.2128 & 0.2133 \\
\hline
\end{tabular}

Note. The dependent variable is Num_BadExp, representing the number of new five-bubble reviews written by experienced reviewers per week for Columns (1) and (2). Focal is a dummy variable that equals 1 if a hotel is in the focal group, and 0 if it is a control hotel. Rating_lag1 is the highest bubble rating score on a hotel's profile page for the previous week. TotalReview lag1 refers to the total number of reviews on a hotel's profile page for the previous week. Specialoffer_lag1 represents the number of times a special offer is posted on a hotel's profile page for the previous week.

Standard errors clustered at the hotel level in parentheses. ${ }^{*} p<0.1,{ }^{* *} p<0.05$, and ${ }^{* * *} p<0.01$

\section{Conclusion}

Our goal is to understand how the Favorite Reviews feature affects user's behavior on a UGC platform in terms of distribution of the volumes of new reviews. We find that the amount of new five-bubble reviews increases significantly after the favorite review has been pinned for the hotels that have the feature, relative to the control group. Our results indicate that the distribution of new reviews with different ratings tends to be distorted toward the more positive extreme. The Favorite Reviews feature might lead to an incentive distortion, which would inflate five-bubble reviews and reduce negative reviews. More importantly, we find that the increment of five-bubble reviews is mainly driven by the new reviewers or new users who registered within 30 days. The reduction on one- or two-bubble reviews, however, is mostly due to existing reviewers who have wrote at least one review before. This would bring a concern of review manipulation by the business owners or managers. This gamification feature seems to hurt the intrinsic motivation of experience users to contribute content. Consequently, the feature may hinder the sustainability of the UGC platform.

WOM is not only important for consumers and business owners, but also important for online UGC platforms such as TripAdvisor, Yelp and Angie's List, whose businesses rely heavily on user reviews. Business owners who do business on those platforms desire more reviews since research has shown that volume of online reviews has a positive impact on product sales. The business owners would purchase value-added products from the platform if they find the products valuable. Consumers, on the other hand, would be attracted by the platform and contribute high-quality content if they get motivated. Therefore, to sustain the businesses, those online UGC platforms would seek methods to encourage users to contribute reviews with good quality and quantity.

This study has important business implications for designing gamification features to motivate users to contribute content online. Our findings suggest that the online platform needs to keep an eye on any new feature it launches and adjust its initiative if an adverse outcome is observed, since the motivation of users, including review writers and review readers, may be affected by a small change. A carefully-designed gamification feature, such as Favorite Reviews feature, may serve as an effective reputation management tool for hotels in the short run. However, as the number of hotels that implement the feature increases, we have a concern that this may jeopardize the credibility of the platform. The hotels also need to think carefully about whether to adopt the feature as it involves a tradeoff between benefit and cost.

In the current study, we do not quantify or differentiate the motivating effect and anchoring effect. In the future, we can investigate whether individuals actually anchor onto the content of the favorite reviews by incorporating text-mining techniques. We can also explore user review histories to find evidence of review manipulation.

\section{References}

[1] Belch, G.E. and Belch, M.A. 2013. "A content analysis study of the use of celebrity endorsers in magazine advertising", International Journal of Advertising, 32(3), pp.369-389.

[2] Mehta, A. 2000. "Advertising Attitudes and Advertising Effectiveness", Journal of Advertising Research, 40 (3), pp. 67-72.

[3] eMarketer.com. 2008. "Online Reviews Sway Shoppers", https://www.emarketer.com/Article/OnlineReviews-Sway-Shoppers/1006404 Last accessed. 04/26/2018. 
[4] TurnTo Report in March. 2017. http://www2.turntonetworks.com/2017consumerstudy [5] Luca, M. and Zervas, G. 2016. "Fake it till you make it: Reputation, competition, and yelp review fraud", Management Science 62(12), pp.3412-3427.

[6] Chevalier, J. A., and Mayzlin, D. 2006. "The effect of word of mouth on sales: Online book reviews", Journal of marketing research, 43(3), pp.345-354.

[7] Litvin, S. W., Goldsmith, R. E., and Pan, B. 2008. "Electronic word-of-mouth in hospitality and tourism management", Tourism management, 29(3), pp.458468.

[8] Bronner, F. and de Hoog, R. 2011, "Vacationers and eWOM : Who Posts, and Why, Where, and What?", Journal of Travel Research, 50 (1), pp. 15-26.

[9] Nielsen's Global Trust in Advertising. http://www.nielsen.com/us/en/insights/news/2012/cons umer-trust-in-online-social-and-mobile-advertisinggrows.html

[10] Xie, K. L., So, K. K. F., and Wang, W. 2017. “Joint effects of management responses and online reviews on hotel financial performance: A data-analytics approach", International Journal of Hospitality Management, 62, pp.101-110.

[11] Ryan, R. M., and Deci, E. L. 2000. "Intrinsic and extrinsic motivations: Classic definitions and new directions", Contemporary educational psychology, 25(1), pp. 54-67.

[12] Deci, E. L., Koestelr, R., and Ryan, R. M. 1999. “A nieta-anualytic review of experiments examining the effects of extrinsic rewards on intrinsic motivation", Psychological Bulletin, 125, pp.627-668.

[13] Khern-am-nuai, W., and Kannan, K. 2014. "Extrinsic versus intrinsic rewards to participate in a crowd context: An analysis of a review platform", Working paper.

[14] Qiao, D., Lee, S. Y., Whinston, A., and Wei, Q. 2017. "Incentive Provision and Pro-Social Behaviors", HICSS.

[15] Cheung, C. M., and Lee, M. K. 2012. "What drives consumers to spread electronic word of mouth in online consumer-opinion platforms", Decision support systems, 53(1), pp.218-225.

[16] Constant, D., Kiesler, S., and Sproull, L. 1994. "What's mine is ours, or is it? A study of attitudes about information sharing", Information systems research, 5(4), pp.400-421.

[17] Toubia, O., and Stephen, A. T. 2013. "Intrinsic vs. image-related utility in social media: Why do people contribute content to twitter?", Marketing Science, 32(3), pp.368-392.

[18] Ma, M., and Agarwal, R. 2007. "Through a glass darkly: Information technology design, identity verification, and knowledge contribution in online communities", Information systems research, 18(1), 42-67.

[19] Wasko MM., and Faraj S. 2005. "Why should I share? Examining social capital and knowledge contribution in electronic networks of practice", MIS Quart. 29(1), pp.35-57.

[20] Chen Y, Harper FM, Konstan J, Li SX. 2010. "Social comparisons and contributions to online communities: A field experiment on MovieLens", Amer. Econom. Rev. 100(4), pp.1358-1398.

[21] Tversky, A., and Kahneman, D. 1974. "Judgment under uncertainty: Heuristics and biases", Science 185(4157), pp.1124-1131.

[22] Furnham, A., and Boo, H. C. 2011. "A literature review of the anchoring effect", The Journal of SocioEconomics, 40(1), pp.35-42.

[23] Wan, Y., and Nakayama, M. 2014. "The reliability of online review helpfulness", Journal of Electronic Commerce Research, 15(3), pp.179.

[24] Adomavicius, G., Bockstedt, J. C., Curley, S. P., and Zhang, J. 2013. "Do recommender systems manipulate consumer preferences? A study of anchoring effects", Information Systems Research, 24(4), pp.956975.

[25] Festinger, L. 1954. A theory of social comparison processes. Human relations, 7(2), pp.117-140.

[26] Shang, R. A., Chen, Y. C., and Huang, C. C. 2012. "A Stage For Social Comparison-The Value Of Information In Virtual Communities", In PACIS, pp. 166.

[27] Buunk, B.P., and Gibbons, F.X. 2007. "Social comparison: The end of a theory and the emergence of a field", Organizational Behavior and Human Decision Processes, 102 (1), pp.3-21.

[28] Wood, J.V. 1989. "Theory and research concerning social comparisons of personal attributes", Psychological Bulletin, 106 (2), pp.231-248.

[29] Lockwood, P., and Kunda, Z. 1997. "Superstars and me: Predicting the impact of role models on the self', Journal of personality and social psychology, 73(1), pp.91. 